\title{
Corneal aberrations after small-incision lenticule extraction versus Q-value-guided laser-assisted in situ keratomileusis
}

\author{
ZhangJun, Zheng Li*, Zhao Xia, Sun Yi, Feng Wei and Yuan Minhui \\ Hangzhou MSK Eye Hospital, No. 384, FengQi Road, Hangzhou, Zhejiang Province, China
}

\begin{abstract}
Aim: To compare the changes in anterior corneal surface aberration characteristics after small-incision lenticule extraction (SMILE) versus Q-value-guided femtosecond laser-assisted in situ keratomileusis (Q-FS-LASIK)

Methods: In this prospective comparative study, 102 patients with myopia and myopic astigmatism were divided between the SMILE and Q-FS-LASIK groups, consisting of 51 patients each. High order aberration (HOA), primary spherical aberration (PSA), and primary coma aberration (PCA) of the central 6 mm region of the anterior corneal surface were quantitatively assessed using pre-and post-operative Sirius scanning, and then statistical analysis with repeated measures analysis of variance.

Results: Both types of surgery were associated with statistically significant increases in post-operative HOA, PSA, and PCA (both groups $\mathrm{P}<0.01$ ). In the SMILE group, the variations in HOA, PSA, and PCA were not statistically significant starting at post-operative week $2(\mathrm{P}>0.05)$. In the Q-FS-LASIK group, the variations in HOA and PCA were not statistically significant starting at post-operative day $1(\mathrm{P}>0.05)$.
\end{abstract}

Conclusion: Both SMILE and Q-FS-LASIK resulted in an increase in HOA, PSA, and PCA at post-operative day 1. However, Q-FS-LASIK introduced lower HOA and showed better stability. Spherical measurement is highly related to PSA.

\section{Introduction}

With the rapid and extensive development of modern corneal refractive surgery, related technologies have been promoted, and new surgical procedures have been developed. Q-value-guided femtosecond laser-assisted in situ keratomileusis (Q-FS-LASIK) is a safe surgical procedure in which lower spherical aberration is introduced [1-4], and it has been a popular procedure for corneal refractive surgery. Small-incision lenticular extraction (SMILE) is a new kind of surgical procedure that avoids flap-related complications [5-6] and is gaining more attention. Both procedures have performed well in studies in all measures of safety, efficacy, and predictability [6-10]. Although some previous studies have compared ocular aberration and visual quality after SMILE and LASIK, anterior corneal surface aberration properties can evaluate the effect of refractive surgery on corneal morphology more accurately and more intuitively and then reflect the influence of refractive surgery on visual quality. The principle will be elaborated in the discussion section. Therefore, the aims of this study were to increase anterior corneal surface aberration parameters to provide a better frame of reference. In addition, and different from earlier research, we used Q-FS-LASIK, which has not previously been compared with SMILE.

In the current study, we measured high order aberration (HOA), primary spherical aberration (PSA), and primary coma aberration (PCA) of the central $6 \mathrm{~mm}$ region of the anterior corneal surface and evaluated the changes in corneal aberration post-operative characteristics.

\section{Methods}

The study and data collection were carried out with approval from Hangzhou MSK Eye Hospital Independent Ethics Committee.
In this prospective, non-randomized study (patients were allocated to either type of surgery via their own wishes) a continuous 102 patients (200 eyes), who met the screening criteria and had myopia or myopia astigmatism underwent either SMILE or Q-FS-LASIK at Hangzhou MSK Eye Hospital between January and November 2015. All participants were informed about the risks and benefits of both procedures and provided written informed consent. Patient inclusion criteria included a sphere plus cylinder measurement of less than $-10.00 \mathrm{D}$ and a cylinder measurement of less than $-5.00 \mathrm{D}$.

\section{Pre-Operative assessments}

Pre-operative assessments included a complete medical and ophthalmological history and a thorough ocular examination, including measurements of uncorrected visual acuity, manifest refraction, best corrected visual acuity, cycloplegic refraction, slit-lamp examination, axial length, gonioscopy, funduscopy, and intraocular pressure. In addition, corneal topography was obtained using a tomography instrument (Sirius; CSO, Florence, Italy).

\section{Measurement of anterior corneal surface aberration}

HOA, PSA, and PCA of the central $6 \mathrm{~mm}$ region of the anterior corneal surface were determined using Sirius. The aberration value

Correspondence to: Zheng Li, Hangzhou MSK Eye Hospital, No. 384, FengQi Road, XiaCheng District, Hangzhou, Zhejiang Province, 310006, China, Tel: 15857189999; Fax: 0571-85068587; E-mail: 164598113@qq.com

Key words: small incision, Q-value guided, femtosecond, aberration

Received: February 15, 2018; Accepted: February 28, 2018; Published: March 03, 2018 
with high quality, high repeatability, and high centrality was used for statistical analysis. High quality was defined as a device signal classification based on the composite index of scheimpflug and keratoscopy images and fixation states. High repeatability was defined as a tangential anterior corneal curvature difference $<0.5 \mathrm{D}$, anterior and posterior elevate difference $<5 \mathrm{um}$. High centrality was defined as a device signal percentage, based on keratoscopy image of more than $90 \%$.

\section{Surgical procedures}

All surgical procedures were performed by a single surgeon. Routine disinfection and surface anesthesia were performed before surgery.

\section{SMILE}

A total of 100 eyes underwent SMILE (VisuMax; Carl Zeiss, Oberkochen, Germany). During the procedure, a cap of $120 \mu \mathrm{m}$, a single side-cut incision with a circumferential length of $2.0 \mathrm{~mm}$ at the 120 -degree position, a side-cut angle of $90^{\circ}$, a $3 \times 3 \mu \mathrm{m}$ point spacing of the lens surface, a $2.5 \times 2.5 \mu \mathrm{m}$ point spacing of the lens side, and a 2 $\times 2 \mu \mathrm{m}$ point spacing of the side cut were created. After a femtosecond laser scan, both the front and back lens surfaces were separated using a micro-separator. The free lens was then removed using micro-forceps.

\section{Q-FS-LASIK}

A total of 100 eyes underwent Q-FS-LASIK with the use of the FS200 femtosecond and EX500 excimer lasers (both from Alcon, Fort Worth, Texas, USA). During flap creation, settings were adjusted to achieve a thickness of $100 \mu \mathrm{m}$, side-cut angle of $90^{\circ}, 8 \times 8 \mu \mathrm{m}$ point spacing of the flap, and $5 \times 3 \mu \mathrm{m}$ point spacing of the side cut. After a femtosecond laser scan, the corneal stroma was ablated with a 0.2 negative adjustment of the $\mathrm{Q}$ value $(6 \mathrm{~mm})$.

\section{Post-Operative care and follow-up}

After surgery, fluorometholone $0.1 \%$, and bromfenac sodium $0.1 \%$ were immediately administered topically, levofloxacin $0.3 \%$ (Cravit; Santen, Osaka, Japan) was administered topically four times a day for one week, and fluorometholone $0.1 \%$ was administered topically six times a day for three weeks, after which the frequency was steadily tapered. Patients were followed, and the Sirius measurements were repeated at post-operative day 1 , week 2 , and months one and three.

\section{Statistical analysis}

All statistical analyses were performed using SPSS 19.0 (SPSS Inc., Chicago, USA). The Student's $t$ test was used to compare pre-operative patient demographics between the two groups. For the purpose of statistical comparisons, visual acuity measurements were converted to logarithms of the minimum angles of resolution (LogMAR) units. Repeated measures analysis of variance was used to assess HOA, PSA, and PCA at different examination points within and between each group. Pre- and post-operative HOA, PSA, and PCA were confirmed did not meet the mauchly's test of sphericity (GreenhouseGeisser $<0.7$ ), Bonferroni test was used for multivariate statistical analysis and comparisons between each group, after degree of freedom Greenhouse-Geisser correction. Pearson correlation coefficients were calculated to evaluate correlations between 3-month changes in HOA, PSA, PCA, and multiple variables, including spherical, cylinder, spherical equivalent (SE), and spherical plus cylinder measurements. The results are expressed as mean $\pm \mathrm{SD}$, and $\mathrm{P}$ values of $<0.05$ were considered statistically significant.

\section{Results}

The study enrolled 52 patients (200 eyes) with the patients divided into two equal groups. Pre-operative patient demographics are summarized in Table 1. All operations were successful, and no serious complications or iatrogenic corneal ectasia were seen during the 3-month post-operative period.

The corneal aberration parameters are summarized in Table 2. The changes in HOA, PSA, and PCA following SMILE and Q-FS-LASIK are shown in Figures 1, 2, and 3, respectively.

In the SMILE group, variations in HOA, PSA, and the PCA were statistically significant $(\mathrm{P}<0.001)$. Bonferroni test showed that HOA, PSA, and PCA at each post-operative time point were statistically significantly higher than the pre-operative values $(\mathrm{P}<0.001)$. However, after the two weeks post-operative timepoint, the differences between each post-operative time point were not significant (Table 3).

In the Q-FS-LASIK group, the variations in HOA, PSA, and PCA were statistically significant $(\mathrm{P}<0.001)$. Bonferroni test showed that HOA, PSA, and PCA at each post-operative time point were statistically significantly higher than the pre-operative value $(\mathrm{P}<0.001)$. However, after the first post-operative day, HOA and PCA differences between each post-operative time point was not significant. PSA was statistically significant between post-operative day 1 day and post-operative months 1 and 3 , and there were no statistically significant differences between the other post-operative time points (Table 4).

The differences in HOA between the SMILE and Q-FS-LASIK groups were statistically significant $(\mathrm{F}=5.33, \mathrm{P}=0.02)$.

The differences in PSA and PCA between the SMILE and Q-FSLASIK groups were not statistically significant $(F=1.45, \mathrm{P}=0.23$ and $F=1.31, \mathrm{P}=0.25$ ).

In both groups, the 3-month changes in HOA and PSA correlated with spherical, SE, and spherical plus cylinder measurements.

In the SMILE group, the 3-month changes in PCA was not correlated with spherical, SE and spherical plus cylinder measurements. Cylinder measurements were not correlated with HOA, PSA, and PCA.

In the Q-FS-LASIK group, the 3-month changes in PCA correlated with spherical, SE, and spherical plus cylinder measurements. Cylinder measurements correlated with PSA (Table 5).

\section{Discussion}

A perfect refractive system is one through which parallel light can pass and in which a wavefront will become an ideal spherical wavefront. The human eye is not a perfect refractive system; therefore, differences between actual and ideal wavefronts will appear. This difference, which is called wavefront aberration [11], is an important index that is used to evaluate irregular corneal morphology and visual quality [12]. There are two main sources of wavefront aberrations in human eyes: 1.) the cornea and 2.) the lens [13]. In the experimental design stage, several reasons led us to choose corneal anterior surface aberrations to evaluate the effect of refractive surgery on corneal morphology and visual quality: 1.) corneal anterior surface aberrations accounts for nearly $80 \%$ of the total aberration of eye ball and significantly influences visual quality $[13,14]$; 2.) corneal refractive surgery mainly modifies the corneal anterior surface shape and does not modifies the lens shape; 3.) compared with the total aberration of eye ball, corneal anterior surface aberrations are less disturbed by tears, pupil size, pupillary center position, and kappa angle [15]. Therefore, corneal 
Table 1. Pre-operative patient demographics

\begin{tabular}{|c|c|c|c|}
\hline Parameter & SMILE & Q-FS-LASIK & t value \\
\hline Age & $24.26 \pm 6.14$ & $25.07 \pm 5.20$ & -1.01 \\
\hline Spherical(D) & $-4.48 \pm 1.22$ & $-4.33 \pm 1.21$ & -0.90 \\
\hline Cylinder(D) & $-0.98 \pm 0.61$ & $-0.86 \pm 0.77$ & -1.27 \\
\hline Best corrected visual acuity & $-0.05 \pm 0.05$ & $-0.04 \pm 0.05$ & -1.73 \\
\hline Uncorrected visual acuity & $1.29 \pm 0.30$ & $1.24 \pm 0.28$ & 0.37 \\
\hline Corneal thickness( $\mu \mathrm{m})$ & $551.68 \pm 26.01$ & $545.98 \pm 28.36$ & 0.21 \\
\hline Optical zone(mm) & $6.44 \pm 0.14$ & $6.40 \pm 0.17$ & 0.27 \\
\hline
\end{tabular}

Table 2. Corneal aberration parameters

\begin{tabular}{|c|c|c|c|c|c|c|c|}
\hline \multirow{2}{*}{ Parameter } & \multirow{2}{*}{ Preoperative } & \multicolumn{4}{|c|}{ Postoperative } & \multirow{2}{*}{ F value } & \multirow{2}{*}{$P$ value } \\
\hline & & 1 day & 2 weeks & 1 month & 3 months & & \\
\hline \multicolumn{8}{|c|}{ High order aberration $(\mu \mathrm{m})$} \\
\hline SMILE & $0.39 \pm 0.08$ & $0.58 \pm 0.18$ & $0.69 \pm 0.19$ & $0.70 \pm 0.16$ & $0.71 \pm 0.17$ & 74.68 & 0.001 \\
\hline Q-FS-LASIK & $0.36 \pm 0.08$ & $0.58 \pm 0.20$ & $0.61 \pm 0.20$ & $0.63 \pm 0.18$ & $0.65 \pm 0.19$ & 45.10 & 0.001 \\
\hline \multicolumn{8}{|c|}{ Primary spherical aberration $(\mu \mathrm{m})$} \\
\hline SMILE & $-0.21 \pm 0.06$ & $-0.33 \pm 0.13$ & $-0.43 \pm 0.13$ & $-0.43 \pm 0.13$ & $-0.45 \pm 0.13$ & 68.85 & 0.001 \\
\hline Q-FS-LASIK & $-0.21 \pm 0.06$ & $-0.34 \pm 0.17$ & $-0.37 \pm 0.16$ & $-0.40 \pm 0.15$ & $-0.43 \pm 0.16$ & 34.05 & 0.001 \\
\hline \multicolumn{8}{|c|}{ Primary coma aberration $(\mu \mathrm{m})$} \\
\hline SMILE & $0.20 \pm 0.08$ & $0.27 \pm 0.15$ & $0.38 \pm 0.19$ & $0.37 \pm 0.18$ & $0.36 \pm 0.20$ & 22.67 & 0.001 \\
\hline Q-FS-LASIK & $0.18 \pm 0.09$ & $0.29 \pm 0.18$ & $0.33 \pm 0.17$ & $0.34 \pm 0.16$ & $0.34 \pm 0.17$ & 18.76 & 0.001 \\
\hline
\end{tabular}

Table 3. Multiple comparisons within SMILE group

\begin{tabular}{|c|c|c|}
\hline \multicolumn{2}{|c|}{ Parameter } & $P$ value \\
\hline \multicolumn{3}{|c|}{ High order aberration } \\
\hline \multirow{4}{*}{ Pre-operative } & Post-operative day 1 & 0.001 \\
\hline & Post-operative week 2 & 0.001 \\
\hline & Post-operative month 1 & 0.001 \\
\hline & Post-operative month 3 & 0.001 \\
\hline \multirow{3}{*}{ Post-operative day 1} & Post-operative week 2 & 0.001 \\
\hline & Post-operative month 1 & 0.001 \\
\hline & Post-operative month 3 & 0.001 \\
\hline \multirow{2}{*}{ Post-operative week 2} & Post-operative month 1 & 0.99 \\
\hline & Post-operative month 3 & 0.99 \\
\hline Post-operative month 1 & Post-operative month 3 & 0.99 \\
\hline \multicolumn{3}{|c|}{ Primary spherical aberration } \\
\hline \multirow{4}{*}{ Pre-operative } & Post-operative day 1 & 0.001 \\
\hline & Post-operative week 2 & 0.001 \\
\hline & Post-operative month 1 & 0.001 \\
\hline & Post-operative month 3 & 0.001 \\
\hline \multirow{3}{*}{ Post-operative day 1} & Post-operative week 2 & 0.001 \\
\hline & Post-operative month 1 & 0.001 \\
\hline & Post-operative month 3 & 0.001 \\
\hline \multirow{2}{*}{ Post-operative week 2} & Post-operative month 1 & 0.99 \\
\hline & Post-operative month 3 & 0.89 \\
\hline Post-operative month 1 & Post-operative month 3 & 0.97 \\
\hline \multicolumn{3}{|c|}{ Primary coma aberration } \\
\hline \multirow{4}{*}{ Pre-operative } & Post-operative day 1 & 0.001 \\
\hline & Post-operative week 2 & 0.001 \\
\hline & Post-operative month 1 & 0.001 \\
\hline & Post-operative month 3 & 0.001 \\
\hline \multirow{3}{*}{ Post-operative day 1} & Post-operative week 2 & 0.001 \\
\hline & Post-operative month 1 & 0.001 \\
\hline & Post-operative month 3 & 0.01 \\
\hline \multirow{2}{*}{ Post-operative week 2} & Post-operative month 1 & 0.99 \\
\hline & Post-operative month 3 & 0.99 \\
\hline Post-operative month 1 & Post-operative month 3 & 0.99 \\
\hline
\end{tabular}


Table 4. Multiple comparisons within Q-FS-LASIK group

\begin{tabular}{|c|c|c|}
\hline \multicolumn{2}{|c|}{ Parameter } & $P$ value \\
\hline \multicolumn{3}{|c|}{ High order aberration } \\
\hline \multirow{4}{*}{ Pre-operative } & Post-operative day 1 & 0.001 \\
\hline & Post-operative week 2 & 0.001 \\
\hline & Post-operative month 1 & 0.001 \\
\hline & Post-operative month 3 & 0.001 \\
\hline \multirow{3}{*}{ Post-operative day 1} & Post-operative week 2 & 0.93 \\
\hline & Post-operative month 1 & 0.36 \\
\hline & Post-operative month 3 & 0.06 \\
\hline \multirow{2}{*}{ Post-operative week 2} & Post-operative month 1 & 0.99 \\
\hline & Post-operative month 3 & 0.74 \\
\hline Post-operative month 1 & Post-operative month 3 & 0.99 \\
\hline \multicolumn{3}{|c|}{ Primary spherical aberration } \\
\hline \multirow{4}{*}{ Pre-operative } & Post-operative day 1 & 0.001 \\
\hline & Post-operative week 2 & 0.001 \\
\hline & Post-operative month 1 & 0.001 \\
\hline & Post-operative month 3 & 0.001 \\
\hline \multirow{3}{*}{ Post-operative day 1} & Post-operative week 2 & 0.70 \\
\hline & Post-operative month 1 & 0.03 \\
\hline & Post-operative month 3 & 0.001 \\
\hline \multirow{2}{*}{ Post-operative week 2} & Post-operative month 1 & 0.83 \\
\hline & Post-operative month 3 & 0.11 \\
\hline Post-operative month 1 & Post-operative month 3 & 0.94 \\
\hline \multicolumn{3}{|c|}{ Primary coma aberration } \\
\hline \multirow{4}{*}{ Pre-operative } & Post-operative day 1 & 0.001 \\
\hline & Post-operative week 2 & 0.001 \\
\hline & Post-operative month 1 & 0.001 \\
\hline & Post-operative month 3 & 0.001 \\
\hline \multirow{3}{*}{ Post-operative day 1} & Post-operative week 2 & 0.81 \\
\hline & Post-operative month 1 & 0.55 \\
\hline & Post-operative month 3 & 0.39 \\
\hline \multirow{2}{*}{ Post-operative week 2} & Post-operative month 1 & 0.99 \\
\hline & Post-operative month 3 & 0.99 \\
\hline Post-operative month 1 & Post-operative month 3 & 0.99 \\
\hline
\end{tabular}

Table 5. Correlation analysis

\begin{tabular}{|c|c|c|c|}
\hline \multicolumn{2}{|c|}{ Parameter } & $R$ value & P value \\
\hline \multicolumn{4}{|c|}{ SMILE } \\
\hline \multirow{4}{*}{ High order aberration } & spherical & -0.36 & 0.001 \\
\hline & cylinder & 0.09 & 0.37 \\
\hline & spherical equivalent & -0.34 & 0.001 \\
\hline & spherical plus cylinder & -0.24 & 0.02 \\
\hline \multirow{4}{*}{ Primary spherical aberration } & spherical & 0.42 & 0.001 \\
\hline & cylinder & 0.01 & 0.98 \\
\hline & spherical equivalent & 0.42 & 0.001 \\
\hline & spherical plus cylinder & 0.32 & 0.001 \\
\hline \multirow{4}{*}{ Primary coma aberration } & spherical & -0.18 & 0.07 \\
\hline & cylinder & 0.19 & 0.07 \\
\hline & spherical equivalent & -0.14 & 0.18 \\
\hline & spherical plus cylinder & -0.07 & 0.50 \\
\hline \multicolumn{4}{|c|}{ Q-FS-LASIK } \\
\hline \multirow{4}{*}{ High order aberration } & spherical & -0.47 & 0.001 \\
\hline & cylinder & 0.12 & 0.23 \\
\hline & spherical equivalent & -0.43 & 0.001 \\
\hline & spherical plus cylinder & -0.36 & 0.001 \\
\hline \multirow{4}{*}{ Primary spherical aberration } & spherical & 0.49 & 0.001 \\
\hline & cylinder & -0.37 & 0.001 \\
\hline & spherical equivalent & 0.37 & 0.001 \\
\hline & spherical plus cylinder & 0.23 & 0.02 \\
\hline \multirow{4}{*}{ Primary coma aberration } & Spherical & -0.29 & 0.01 \\
\hline & Cylinder & 0.02 & 0.83 \\
\hline & spherical equivalent & -0.28 & 0.01 \\
\hline & spherical plus cylinder & -0.25 & 0.01 \\
\hline
\end{tabular}




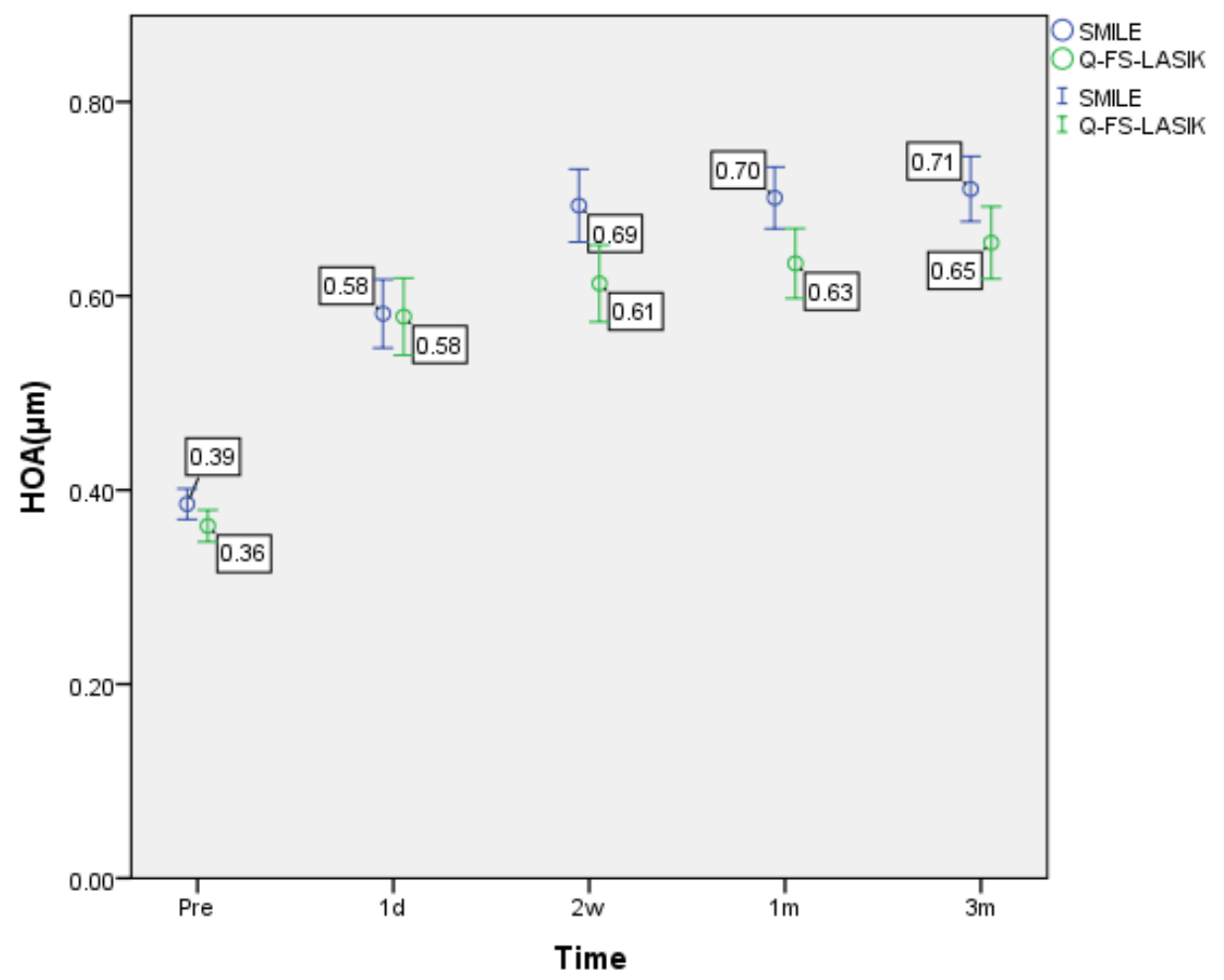

Figure 1. HOA changes following SMILE and Q-FS-LASIK. Changes in HOA over time. Bars represent standard deviations. The differences in HOA between the SMILE and Q-FS-LASIK groups were statistically significant.

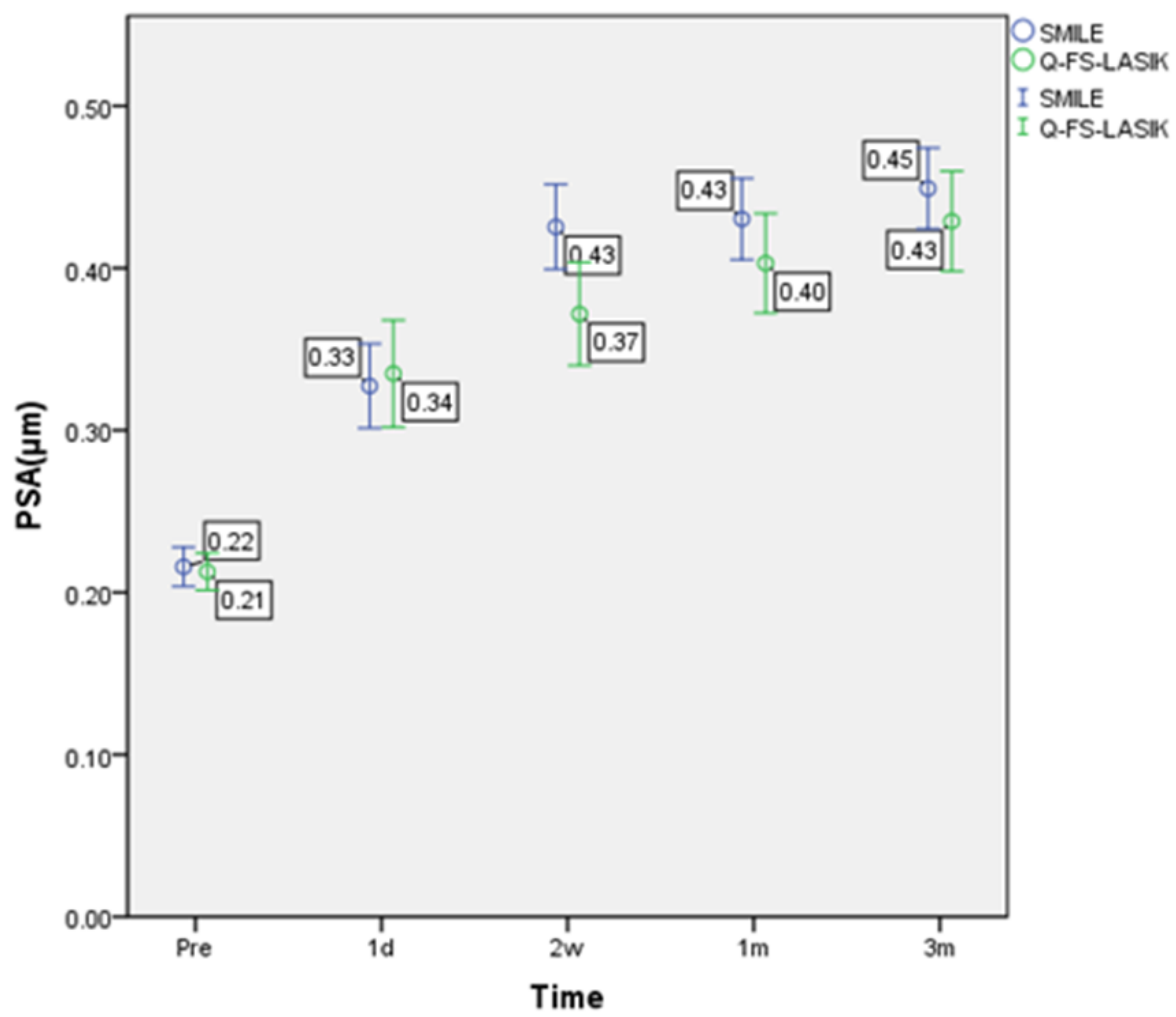

Figure 2. PSA changes following SMILE and Q-FS-LASIK. Changes in PSA over time. Bars represent standard deviations. The differences in PSA between the SMILE and Q-FS-LASIK groups were not statistically significant. 


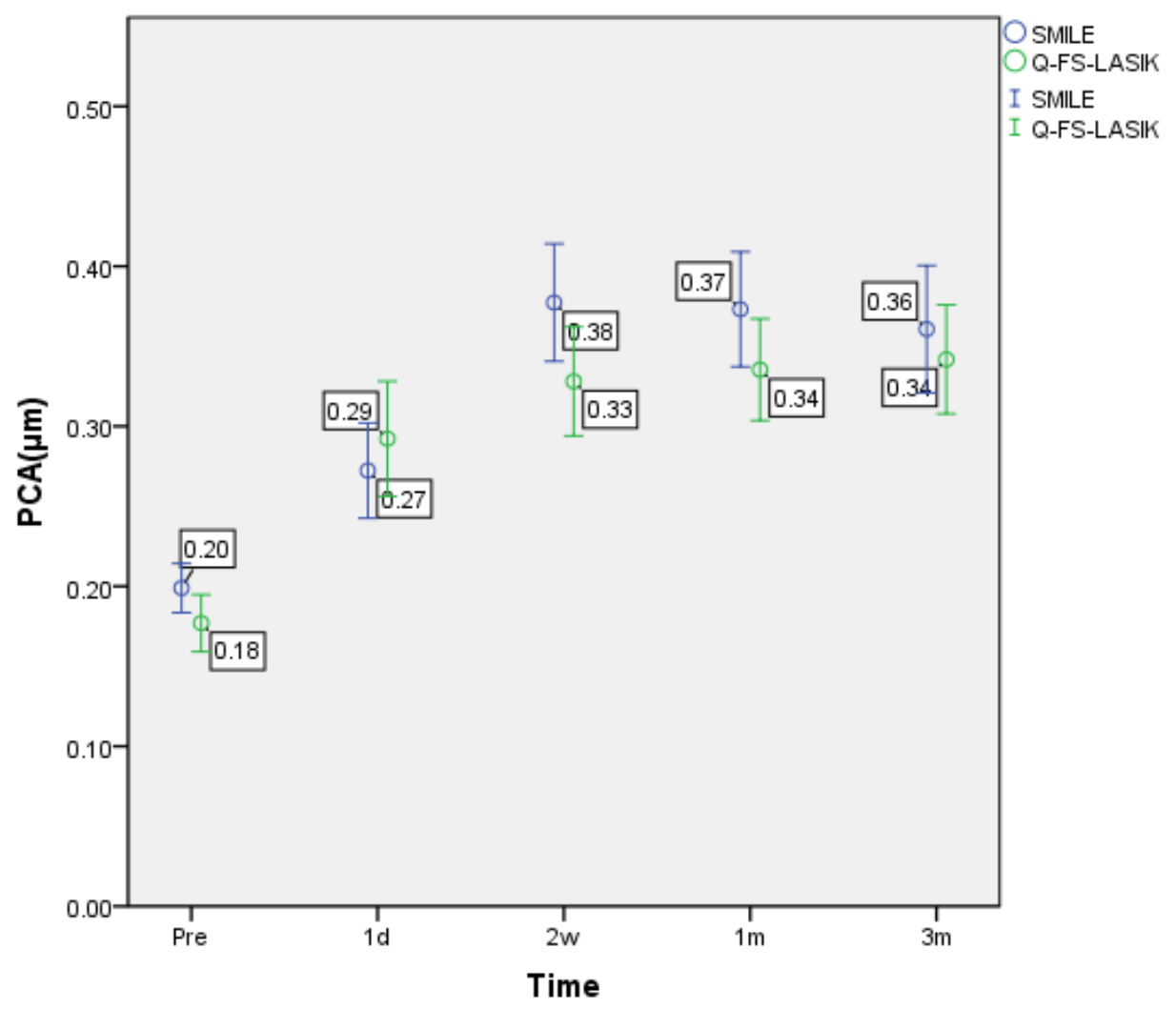

Figure 3. PCA changes following SMILE and Q-FS-LASIK. Changes in PCA over time. Bars represent standard deviations. The differences in PCA between the SMILE and Q-FS-LASIK groups were not statistically significant.

anterior surface aberrations have better repeatability and accuracy; 4.) although low order aberrations have greater impact, they can be adjusted and eliminated with a nomogram according to surgeon's experience. Therefore, the reference value of low order aberrations is not high and does not match the statistical analysis; and 5.) in the Zernike high order aberration chart, relatively low order primary coma and relatively central axial position primary spherical aberrations have the most obvious influence on visual quality [16-17]. Therefore, by analyzing the changes in HOA, PSA and PCA of the corneal anterior surface, the effects of refractive surgery on corneal morphology and visual quality can be more accurately and intuitively evaluated.

The results of this study suggest that high order wavefront aberrations increased by post-operative day 1 following either SMILE or Q-FS-LASIK. This shows that both procedures affected regular corneal morphology and visual quality, especially in highly spherical patients. Consistent with other studies, greater high order wavefront aberrations increased the relative risk of symptoms [18-19].

In the current study, we also found that high order wavefront aberrations were very stable after two post-operative weeks following either SMILE or Q-FS-LASIK. Furthermore, Q-FS-LASIK achieved stability earlier and introduced less high order wavefront high order aberrations. This indicated that Q-FS-LASIK has better stability and better visual quality. Possible causes are analyzed below: 1.) compared with only one-time separation of corneal stroma separated of Q-FSLASIK, two-times corneal stroma separated of SMILE causes more obvious corneal irritation symptoms [20]. Therefore, the cornea needs a relatively long time to completely heal and to allow any edema to subside; 2.) during the SMILE surgery, removal of the lens results in negative capsular pressure on which additional traction on the anterior and posterior stroma surfaces may affect the primary lamellar structure of the cornea; 3.) if only the size of the side cut is considered, Q-FSLASIK's larger side cut may introduce higher wavefront high order aberrations. However, after removing a large number of corneal stroma, compared to corneal cap of SMILE, the corneal flap of Q-FSLASIK should have a better fit with the remaining corneal stroma; 4.) compared to the femtosecond laser blasting, the ablation done by the excimer laser can yield a smoother surface, which need a relatively shorter repair time [21]; and 5.) under the same diopter, SMILE consumes more corneal tissue, which make the change of corneal shape more obvious because SMILE needs to remove a layer of ineffective stroma. Our study had several limitations: 1.) the sample size of our study population was relatively small; 2.) other surgical procedures were not considered as independent factors or control groups; 3.) the non-randomized method of treatment allocation resulted yields a slight imbalance in some baseline patient characteristics between both groups. Selected bias was at least partially offset by having each patient select surgical procedure, however it could not be completely ruled out; and 4.) the study follow-up was limited to three months, which does not rule out the possibility of subsequent regression. Further studies are needed to elucidate long-term aberrations changes.

In summary, both SMILE and Q-FS-LASIK were associated with increases in wavefront high order aberrations. Q-FS-LASIK introduced lower high order aberrations, maintained better visual stability and achieved better visual quality. It may be related to intra-operative corneal irritation, corneal flap or corneal cap tension, stroma removal thickness, remaining stroma smoothness, and healing. A larger sample size and longer follow-up are needed for additional studies. 


\section{Acknowledgment}

\section{Foundation}

Supported by Zhejiang Medical and Health Science and Technology Project (No. 2018267858)

Supported by Hangzhou Science and Technology Development Committee (No. 20140633B44)

\section{References}

1. Amigó A, Bonaque-González S, Guerras-Valera E (2015) Control of Induced Spherical Aberration in Moderate Hyperopic LASIK by Customizing Corneal Asphericity. $J$ Refract Surg 31: 802-806. [Crossref]

2. Goyal JL, Garg A, Arora R, Jain P, Goel Y (2014) Comparative evaluation of higherorder aberrations and corneal asphericity between wavefront-guided andaspheric LASIK for myopia. J Refract Surg 30: 777-784. [Crossref]

3. Pajic B, Vastardis I, Pajic-Eggspuehler B, Gatzioufas Z, Hafezi F (2014) Femtosecond laser versus mechanical microkeratome-assisted flap creation for LASIK: a prospective, randomized, paired-eye study. Clin Ophthalmol 8: 1883-1889. [Crossref]

4. Somani S, Tuan KA, Chernyak D (2004) Corneal asphericity and retinal image quality: a case study and simulations. J Refract Surg 20: S581-585. [Crossref]

5. Ganesh S, Brar S, Arra RR (2018) Refractive lenticule extraction small incision lenticule extraction: A new refractive surgery paradigm. Indian J Ophthalmol 66: 1019. [Crossref]

6. Yan H, Gong LY, Huang W, Peng YL (2017) Clinical outcomes of small incision lenticule extraction versus femtosecond laser-assisted LASIK for myopia: a Metaanalysis. Int J Ophthalmol 10: 1436-1445. [Crossref]

7. Shetty R, Francis M, Shroff R, Pahuja N, Khamar P, et al. (2017) Corneal Biomechanical Changes and Tissue Remodeling After SMILE and LASIK. Invest Ophthalmol Vis Sci 58: 5703-5712. [Crossref]

8. Liu M, Chen Y, Wang D, Zhou Y, Zhang X, et al. (2016) Clinical Outcomes After SMILE and Femtosecond Laser-Assisted LASIK for Myopia and Myopic Astigmatism: A Prospective Randomized Comparative Study. Cornea 35: 210-216. [Crossref]

9. Chansue E, Tanehsakdi M, Swasdibutra S, McAlinden C (2015) Safety and efficacy of VisuMax ${ }^{\circledR}$ circle patterns for flap creation and enhancement following small incision lenticule extraction. Eye Vis (Lond) 2: 21. [Crossref]
10. Kamiya K, Shimizu K, Igarashi A, Kobashi H (2014) Visual and refractive outcomes of femtosecond lenticule extraction and small incision lenticule extraction for myopia. Am J Ophthalmol 157: 128-134. [Crossref]

11. Zohrabi M, Cormack RH, Mccullough C, Supekar OD, Gibson EA, et al. (2017) Numerical analysis of wavefront aberration correction using multielectrode electrowetting-based devices. Opt Express 25: 31451-31461. [Crossref]

12. Nakajima M, Hiraoka T, Hirohara Y, Oshika T, Mihashi T (2015) Verification of the lack of correlation between age and longitudinal chromatic aberrations of the human eye from the visible to the infrared. Biomed Opt Express 6: 2676-2694. [Crossref]

13. Atchison DA, Suheimat M, Mathur A, Lister LJ, Rozema J (2016) Anterior Corneal, Posterior Corneal, and Lenticular Contributions to Ocular Aberrations. Invest Ophthalmol Vis Sci 57: 5263-5270. [Crossref]

14. Wan XH, Li SM, Xiong Y, Liang YB, Li J, et al. (2014) Ocular monochromatic aberrations in a rural Chinese adult population. Optom Vis Sci 91: 68-75. [Crossref]

15. Safarzadeh M, Nasiri N (2016) Anterior segment characteristics in normal and keratoconus eyes evaluated with a combined Scheimpflug/Placido corneal imaging device. J Curr Ophthalmol 28: 106-111. [Crossref]

16. Bühren J, Nagy L, Yoon G, MacRae S, Kohnen TH (2010). The effect of the asphericity of myopic laser ablation profiles on the induction of wavefront aberrations. Invest Ophthalmol Vis Sci 51: 2805-2812. [Crossref]

17. Calvo R, McLaren JW, Hodge DO, Bourne WM, Patel SV (2010) Corneal aberrations and visual acuity after laser in situ keratomileusis: femtosecond laser versus mechanical microkeratome. Am J Ophthalmol 149: 785-793. [Crossref]

18. Sharma M, Wachler BS, Chan CC (2007) Higher order aberrations and relative risk of symptoms after LASIK. J Refract Surg 23: 252-256. [Crossref]

19. Sekundo W, Kunert KS, Blum M (2011) Small incision corneal refractive surgery using the small incision lenticule extraction (SMILE) procedure for the correction of myopia and myopic astigmatism: results of a 6-month prospective study. Br J Ophthalmol 95: 335-339. [Crossref]

20. Shetty R, Francis M, Shroff R, Pahuja N, Khamar P (2017) Corneal Biomechanical Changes and Tissue Remodeling After SMILE and LASIK. Invest Ophthalmol Vis Sci 58: 5703-5712. [Crossref]

21. Weng S, Liu M, Yang X, Liu F, Zhou Y, et al. (2018) Evaluation of Human Corneal Lenticule Quality After SMILE with Different Cap Thicknesses Using Scanning Electron Microscopy. Cornea 37: 59-65. [Crossref]

Copyright: (C2018 Jun Z. This is an open-access article distributed under the terms of the Creative Commons Attribution License, which permits unrestricted use, distribution, and reproduction in any medium, provided the original author and source are credited. 